\title{
Trajectory Planning and Walking Pattern Generation of Humanoid Robot Motion
}

\author{
Saeed Abdolshah*, Majid Abdolshah**, S.H.Tang*** \\ * School of Mechanical Engineering, Sharif University of Technology \\ ** Faculty of New Sciences \& Technologies, University of Tehran \\ *** Department of Mechanical and Manufacturing Engineering, Faculty of Engineering, Universiti Putra Malaysia
}

\begin{tabular}{l} 
Article Info \\
\hline Article history: \\
Received Feb 9, 2015 \\
Revised Apr 25, 2015 \\
Accepted May 12, 2015 \\
\hline
\end{tabular}

\section{Keyword:}

Balance

Humanoid Robot

Kinematics

Trajectory Generation

\begin{abstract}
Walking trajectory generation for a humanoid robot is a challenging control issue. In this paper, a walking cycle has been recognized considering human motion, and nine simple steps were distinguished in a full step of walking which form motion trajectory, and generate a simplified ZMP motion formulation. This system was used in humanoid robot simulation motion and is achievable easily in walking steps of robot. A minimum DOFs humanoid robot has been considered and geometrical relationships between the robot links were presented by the Denavit-Hartenberg method. The inverse kinematics equations have been solved regarding to extracted ZMP trajectory formula, and constraints in different steps. As a result; angular velocity, acceleration and power of motors were obtained using the relationships and Jacobin. At each step, extracted data were applied on simulated robot in Matlab, and Visual Nastran software. Zero moment point trajectory was evaluated in simulation environment.
\end{abstract}

Copyright $\odot 2015$ Institute of Advanced Engineering and Science. All rights reserved.

\section{Corresponding Author:}

Saeed Abdolshah,

School of Mechanical Engineering,

Sharif University of Technology,

Email: abdolshah@alum.sharif.edu

\section{INTRODUCTION}

Motion generation of different robots such as hexapod robots [1,2], four-legged robots [3], and humanoid robots is an essential issue in their control process. The generation of stable humanoid robot motion requires computations of joint trajectories. Several techniques [4, 5, $6 \& 7]$ were proposed to compute stable joint trajectories for walking. Most of advanced humanoid robots have more degrees of freedom (DOF) than the constraints imposed through the balance criterion. Therefore, motions generated solely from the balance criterion adopted in the previous approaches may not yield natural motions since the unspecified DOF are left unutilized. Moreover, planning trajectory may become very complex for a complicated motion such as dancing [8].

On the other hand, motion-captured data from human performers are used to drive animated characters [9]. Obviously, this technique can be used to ease the generation of complicated movements as well as providing motion to the unutilized DOF and yielding natural movement for a humanoid robot. In computer animation and film-making industry, the director can reuse the motion database by manually editing them.

A humanoid robot platform should have expandability of its mechanical structure [10], electronic hardware, and software for the introduction of new elemental functions. With the anticipated widespread use of humanoid robots in the human world, users may hope that they can reconfigure their humanoid robots to suit their needs and preferences as easily as they can custom their personal computers. 
The first step in generating humanoid robots motion is statically and dynamically simulation [11] and trajectory design [12]. Most of the methods are too long and a combination of a few approaches like making a trajectory using learning algorithms [13] or neural network [14] is time consuming, however; there are some data in comparison of different types of walking styles which can help trajectory generation [15].

In this paper a primary walking cycle has been recognized via inspecting on human motion, consequently nine steps were categorized in a full step of walking. Using FSR sensors a Zero Moment Point trajectory was designed via a trajectory generator and simplified as a formula of human ZMP trajectory which was used in humanoid robot motion. A minimum DOFs of humanoid robot has been considered and geometrical relationship between the robot links were presented by the Denavit-Hartenberg method. As a result; velocity, acceleration and power of motors have been evaluated. At each step, robot would be moved in Matlab software, and zero moment point trajectory was also obtained.

\section{HUMANOID ROBOTS BALANCE THEORIES}

There are several theories for holding humanoid robots in balance. One of the old theories is balancing by calculating center of gravity (COG) point. In this theory, projection of robots' center of mass should always be in the support polygon. In another method, ZMP [16] is used. It specifies the point which dynamic reaction force at the contact of the foot with the ground does not produce any moment. In real human walking motion, the point ZMP is placed inside the allowable balanced area [17]. For calculating the center of mass point, Equation 1 is used:

$$
\left\{\begin{array}{l}
x_{C O G} \\
y_{C O G} \\
z_{C O G}
\end{array}\right\}=\frac{1}{\sum_{i=1}^{n} m_{i}}\left\{\begin{array}{l}
\sum_{i=1}^{n} m_{i} x_{i} \\
\sum_{i=1}^{n} m_{i} y_{i} \\
\sum_{i=1}^{n} m_{i} z_{i}
\end{array}\right\}
$$

Where $x_{C O G}, y_{C O G}, z_{C O G}$ are the positions of the mass center of the robot; and $x_{i}, y_{i}, z_{i}$ are thepositions of the mass center of the link $\mathrm{i} ; m_{i}$ is the mass of the link $\mathrm{i}$ and $\mathrm{n}$ is the total number of the components of the robot.

Zero Moment Point (ZMP) is achieved by finding a point as (x, y) which the sum of all inertial torques around it, is zero. Since the projection of ZMP is in the area of the feet, the robot will keep its balance. Equation 2 is the ZMP formulation; however complexity of ZMP calculation, forced the researchers to use a simple formulation which uses the FSR sensor values which were implanted under the sole of legs, as mentioned before.

$$
\left\{\begin{array}{c}
x_{z m p} \\
y_{z m p}
\end{array}\right\}=\frac{1}{\sum_{i=1}^{n} m_{i}\left(\ddot{z}_{i}-g_{z}\right)-\sum_{k} F_{z, k}} \times\left\{\begin{array}{c}
\sum_{i=1}^{n}\left(m_{i}\left(\ddot{z}_{i}-g_{z}\right) x_{i}-m_{i}\left(\ddot{x}_{i}-g_{x}\right) z_{i}\right. \\
\left.-J_{y, i} \ddot{\phi}_{y, i}\right)+\sum_{k}\left(z_{k} F_{x, k}-x_{k} F_{z, k}\right) \\
\sum_{i=1}^{n}\left(m_{i}\left(\ddot{z}_{i}-g_{z}\right) y_{i}-m_{i}\left(\ddot{y}_{i}-g_{y}\right) z_{i}\right. \\
\left.-J_{x, i} \ddot{\phi}_{x, i}\right)+\sum_{k}\left(z_{k} F_{y, k}-y_{k} F_{z, k}\right)
\end{array}\right\}
$$

Where $J_{y, i}, J_{x, i}$ denote the moment of inertia in the $\mathrm{x}$ and y-direction of link i; $\phi_{y, i}, \phi_{x, i}$ denote the pitch angle of link i; $F_{y, k}, F_{z, k}$ are the $\mathrm{y}$ and $\mathrm{z}$-direction of the external force and $g_{x}, g_{y}, g_{z}$ are parts of the gravity acceleration.

Attentive to researches made in this field $[18,19]$ if the points mentioned (COG, ZMP) would be placed in the stable area the robot would be kept in balance. This area is gained from the balance equations 
which is a Convex Hull of the connection points between the feet and ground (convex hull is an area with certain points and minimum sides) (Figure 1).

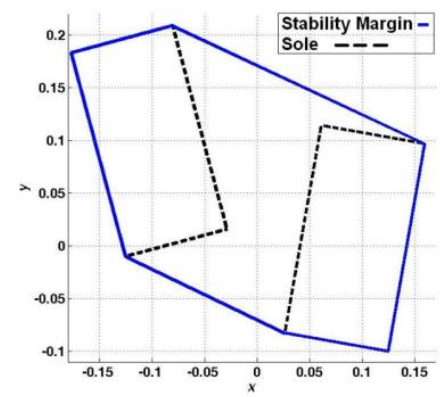

Figure 1. Stability region in arbitrary state of feet

\section{HUMANOID ROBOTS MODEL AND TRAJECTORY GENERATION}

\subsection{Degrees of Freedom}

In this part we have simulated a robot with human like motion. We could define 23 degrees of freedom for body by reviewing the human motion, skeleton of body and counting out the extreme degrees of freedom of each joint. But to make the control easier, and by reviewing the complications of building, robot control and analysis, a robot model with 12 degrees of freedom was selected (which is the minimum degrees required (Figure 2)). Motion of human body could be divided into two components, upper and lower bodies. Since humanoid legs have high degrees of freedom for human-like walking, it is difficult to use their dynamics to design controller and to analyze stability.

Therefore, we will simplify the walking related dynamics of bipedal robot as the equation of motion. The major rule of lower body in motion can be abstracted in 10 degrees of freedom ( 5 degrees of freedom in each leg), 2 degrees at hip, 1 degree at knee and 2 degrees at wrist. This was done so that the robot motion could be closest to human legs movement. To prevent model complication, only 2 degrees of freedom was placed on the waist to help robot for holding in balance. Two degrees of freedom at the waist can be lithe in build. The lither the upper body is, the smoother its gait is. Moreover, the moment generated in the yaw axis of robot can be suppressed by using waist motion. This compensation was applied for high-speed walking.

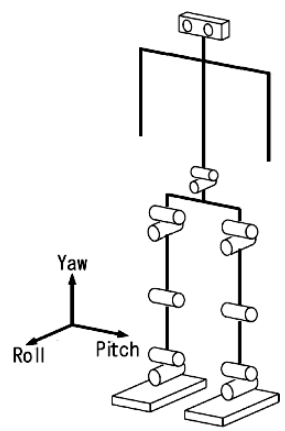

Figure 2. Schematically model of robot with 12 DOF

\subsection{Geometrical Model}

Various ways exist for creating a geometrical model for a robot. One of commonest ways is the Denavit-Hartenberg method. In using this method in the humanoid robots, some notes must be considered. At first, the global coordination which is inside the legs area was transferred to under the sole of leg which the $\mathrm{X}$-direction of this new coordination is in direction of global Z-direction, so the coordination must be rotated -90 degrees around $\mathrm{Y}$ and $\mathrm{X}$ directions of global coordination, then the transmission of the coordination was done to $(0.075,-0.04,0)$ which was the point exactly under the sole of leg. Matrix $\mathrm{T}$ was defined to do the job which is seen in equation 3,4 and Figure 3 shows the transfer. 


$$
\begin{aligned}
& T=\left[\begin{array}{cccc}
\cos (\beta) & \sin (\beta) * \sin (\alpha) & \sin (\beta) * \cos (\alpha) & r_{x} \\
0 & \cos (\alpha) & -\sin (\alpha) & r_{y} \\
-\sin (\beta) & \cos (\beta) * \sin (\alpha) & \cos (\beta) * \cos (\alpha) & r_{z} \\
0 & 0 & 0 & 1
\end{array}\right] \\
& T(1)=\left[\begin{array}{cccc}
0 & 1 & 0 & 0.075 \\
0 & 0 & 1 & -0.04 \\
1 & 0 & 0 & 0 \\
0 & 0 & 0 & 1
\end{array}\right] \quad \begin{array}{cc}
r_{x}=0.075 & \alpha=-90 \\
r_{y}=-0.04 & \beta=-90 \\
r_{z}=0 &
\end{array}
\end{aligned}
$$
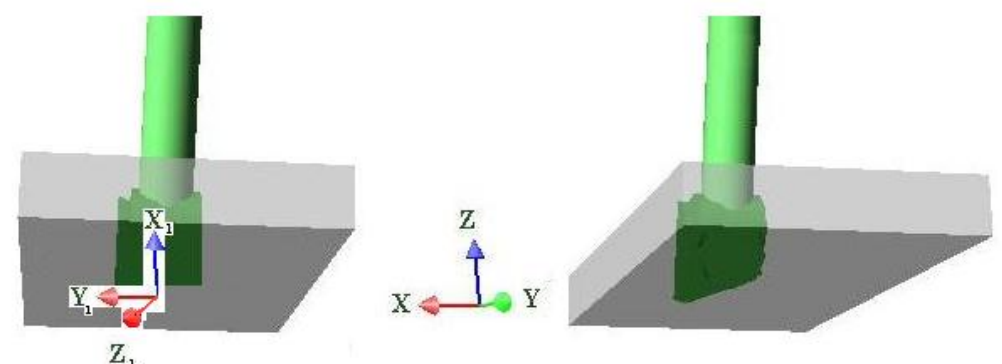

Figure 3. Transferring the coordination to under the sole of leg

At the second step the coordination of sole should be transferred to the wrist, as the wrist has two motors, two stages were considered at first, the first motor rotates around X-direction so the coordination direction was fixed and Denavit-Hartenberg matrix was used like equation 5.

$$
\begin{aligned}
\theta(2) & =0 . \\
T(2) & =\left[\begin{array}{lllc}
1 & 0 & 0 & 0.02 \\
0 & 1 & 0 & 0 \\
0 & 0 & 1 & 0 \\
0 & 0 & 0 & 1
\end{array}\right]
\end{aligned}
$$

At next step, just a rotation was considered to change the $\mathrm{X}$-direction rotating motor coordination to Y-direction rotating motor coordination, also the leg was bowed 15 degrees to keep the initial balance of robot, so the rotation matrix is like equation 6.

$$
\begin{aligned}
\theta(3) & =15 \\
T(3) & =\left[\begin{array}{cccc}
0.9659 & -0.2588 & 0 & 0.01 \\
0 & 0 & -1 & 0 \\
0.2588 & 0.9659 & 0 & 0 \\
0 & 0 & 0 & 1
\end{array}\right]
\end{aligned}
$$

The next step was transferring the coordination to the knee. All the steps were done like this and $\mathrm{T}(1)$ to $\mathrm{T}(11)$ was produced from the right leg to the left leg, $\mathrm{T}(12)$ and $\mathrm{T}(13)$ were generated from the hip to upper body of robot.

In fact, one point of robot is fixed as reference and the relationship between links of robot is stated based on it. This fixed point was selected regarding to the type of motion. For example, when the robot is moving, in each motion, one of the legs will be fixed like a refuge and that point is on sole of fixed leg. By 
selecting fixed leg and putting coordinate system on the sole of this foot, by regarding to universal coordinate system, placed system of other links was selected on basis of Denavit-Hartenberg method. These systems placed on joints of any links that were the same as robot's motor.

Due to Denavit-Hartenberg method which has been written for a kinematical continuum, therefore in hip joint of fixed leg that belongs to two kinematical continuums (continuum of feet, continuum of fixed leg - upper part of body), two coordinate systems were placed, one by regarding to first joint of upper part and another regarding to first joint of another leg.

\subsection{Onilne motion pattern generation}

The humanoid robot consists of rigid links that are connected to several revolute joints, which permit relative motion of neighboring link structure. Transition motion in humanoid robots would be restricted within joint angles and kinematics configuration. However, it is difficult to define a motion pattern. Toward this problem, we tried to generate humanoid robot motion based on analysis of human motion. Such a technique also has the advantage to generate tasks that adapt to environments or conditions for a humanoid robot. The development method was described by the posture of actual humans.

In order to make a special kind of motion such as walking, phases should be joined together. At this stage, we have tried to make some different patterns like moving left leg, moving right leg and so on, which can also ensure us about the robot's stability, the summation of these motions make different motions.

Upon the investigation on human motion, we have generated a complete walking cycle consists of nine phases. Height and length step were fed into the pattern generator. Pattern generator creates the nine steps pattern, and proper trajectory was generated (Figure 4). The results of this process illustrated the projection of ZMP of a human in the stable polygon. To determine the ZMP trajectory, we have used 4 FSR sensors and calculated the ZMP with the equation 7 in the simulation area.

$$
P=\frac{\sum_{i=1}^{4} f_{i} r_{i}}{\sum_{i=1}^{4} f_{i}}
$$

Where forces $f_{i}$ applied on sensor positions and ri is the total distance of sensors from the origin. Our investigations showed that ordinary human ZMP projection has a light sinusoidal curve which in vertical movements has a range about 24 centimeters. To prepare a simple walking method, we can suppose the ZMP trajectory is steady with sinusoidal formula in range of $4^{*}$ pi for a full step, scaled in $200 \mathrm{~cm}$. For simplification we have used this sinusoidal formula to design the trajectory of robot.

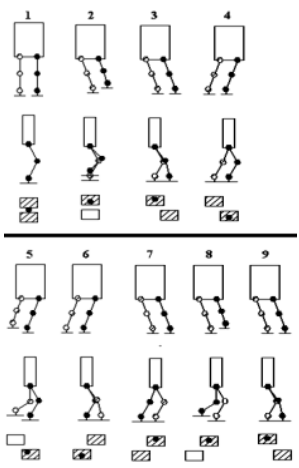

Figure 4. Algorithm of motion

\subsection{Motion steps}

The extracted motion phases from human pattern were applied in this section. The DenavitHartenberg method was used for deriving the relations of the 12 degrees of freedom robot. At the global form for solving the motion equations we have 12 unknown quantities in each step of walking. To gain these 12 unknowns in different movement phases from a set of constraints such as symmetry, constant length of two 
joints of each side of hip, movement direction, and place of extracted ZMP and COG point of human subject was used. Inverse kinematics approach was used to solve the problems.

The equations derived, were mostly nonlinear and in some cases it would be close to singularity state. So other than using the nonlinear equation solving techniques in inverse kinematics, we have used singular value decomposition algorithm. By solving these equations the amount of different variation joint angles for each step was obtained. For calculating the angular velocity, the linear trajectory generation with parabolic limits have been used.

As an example, at the first phase, the robot should lift one of the legs, to prevent falling robot, fist the projection of ZMP and COG should be placed in convex hull, so the projection of ZMP and COG should be transferred to one of the legs, therefore the robot have to lean on one of the legs, to implement this phase just 4 motors- two in wrists and two in hip- were used. Regarding to constraints and ZMP or COG positions, equations have been solved. In other phases some motors have been considered 'off' and other motions have been estimated by equations.

\section{RESULTS}

Considering the humanoid robot motion trajectory, and then obtaining the actuators' motion using kinematics formulas resulted in velocity and acceleration estimation of each link. Then, by using Jacobian, the amount of force and torque of each link was calculated. In Figure 5, the angular velocity, torque and power of left hip motor (x-direction) and right knee motor are presented.

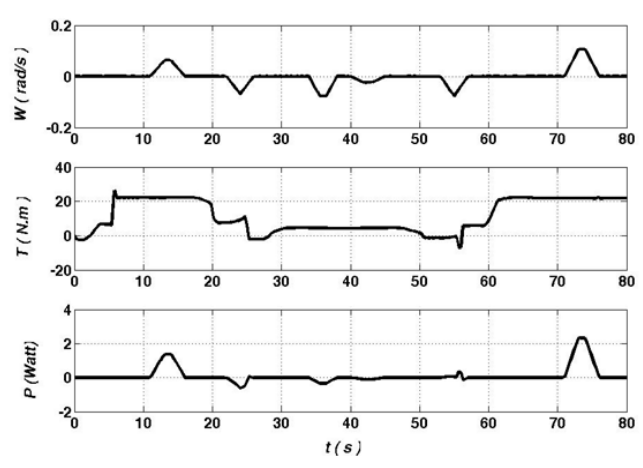

(a)
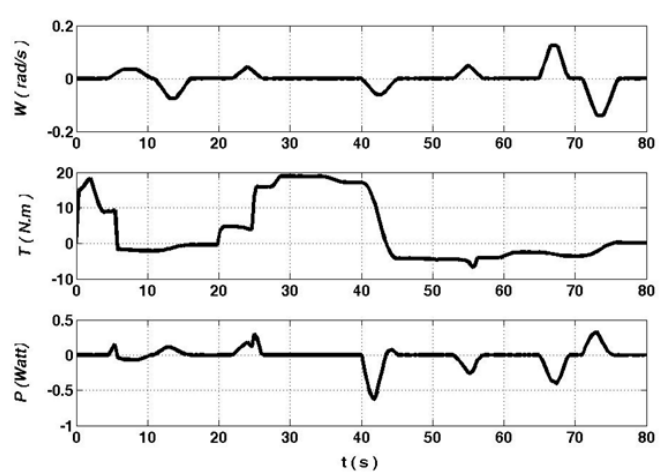

(b)

Figure 5. (a) Properties of left hip motor (x- direction), (b) Properties of right knee motor

The motion of robot was programmed in Matlab software regarding to explanation mentioned earlier. Hence, the motion function of each step was estimated based on Denavit-Hartenberg method and generated pattern regarding to ZMP motion formula, then equations were solved and the robot was moved in Matlab environment.

In Figure 6, the motion of robot from side view and perspectives view are presented. Moreover, the trajectory of ZMP movement in all steps was achieved and is presented in Figure 7.
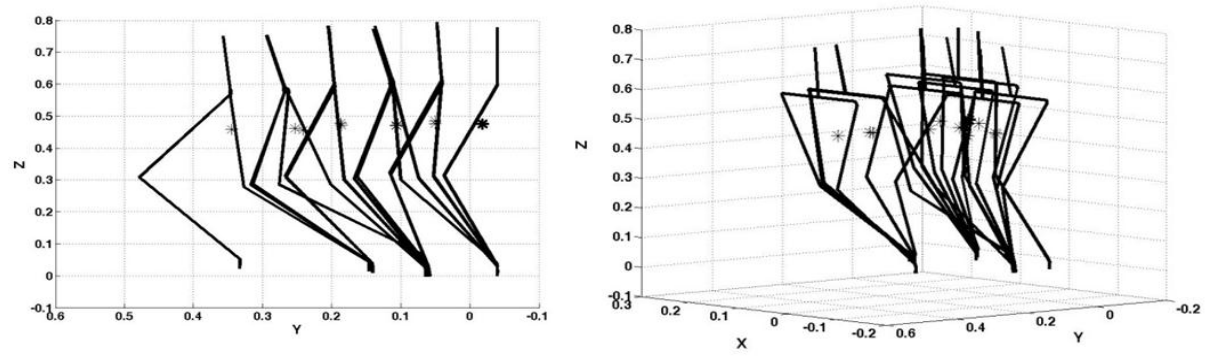

Figure 6. Motion of robot in Matlab software 


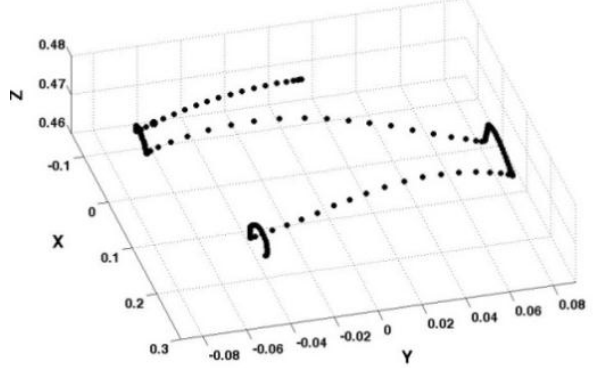

Figure 7. ZMP trajectory of motion of robot in Matlab

For moving the robot in the simulated area, the angular velocity signals calculated for the electro motors and were sent to the Visual Nastran software, and then the simulated robot moved in nine extracted steps (Figure 8).

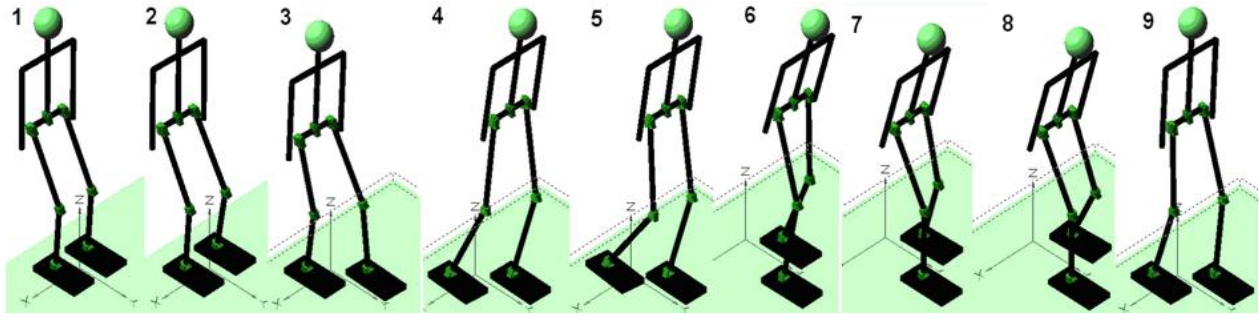

Figure 8. Motion of robot in Visual Nastran software

\section{CONCLUSION}

In this paper the motion of humanoid robot with 12 degrees of freedom was analyzed and the ZMP trajectory was designed simply with a formula via humanoid pattern. Based on research on the human skeleton, the least number of degrees of freedom was selected. Researching on the human motion by a trajectory generator, the motion of human was extracted and ZMP trajectory formulated to be used in humanoid robots motion. The geometrical model of robot was taken out using the Denavit-Hartenberg method. Combining extracted motion and ZMP formula with Denavit-Hartenberg method, motion of robot was obtained and the robot was moved in simulated environment, the results were extracted.

Analyzing the results gives a method which makes the trajectory generation very easier. On the other hand, we must pay attention that gaining an optimized trajectory is very complicated, because other than making a minimum target function for a non-bound mechanism with high degrees of freedom, the robot has to be able to keep its balance. Hence, we need a primary trajectory which could be gained with a reasonable method and should be simple. In addition, gaining control algorithms for usage of robot transformation function in different surfaces is very complicated which mostly is not inspected and it is preferred to use the new methods for the control which primary trajectory design by this method is the necessity to start. Also human subject pattern of walking on uneven surfaces or running can be used for humanoid robots as the future work.

\section{ACKNOWLEDGEMENTS}

At the end we would like to thank the principal of Semnan science and technology center, Mr. Adab, Eng., Iranfanavaran group and Rouyesh research center of Semnan University, and Dr. M.M.Khatibi who cooperated with us in the research process.

\section{REFERENCES}

[1] Addie Irawan., Tan Yee Yin., "Optimizing Hexapod Robot Reconfiguration using Hexa-Quad Transformation" IAES International Journal of Robotics and Automation (IJRA), vol 3, No 2, June 2014. 
[2] Abhijit Mahapatra., Shibendu Shekhar Roy., Dilip Kumar Pratihar, "Modeling and Simulation of Wave Gait of a Hexapod Walking Robot: A CAD/CAE Approach" IAES International Journal of Robotics and Automation (IJRA), vol 2, No 3, September 2013.

[3] Saurav Agarwal., Abhijit Mahapatra., Shibendu Shekhar Roy., "Dynamics and Optimal Feet Force Distributions of a Realistic Four-legged Robot" International Journal of Robotics and Automation (IJRA), vol 1, No 4, June 2012.

[4] Ude, A, Atkeson., C.G., Riley., M., "Planning of joint trajectories for humanoid robots using B-spline wavelets," Robotics and Automation, 2000, Proceedings. ICRA '00. IEEE International Conference on , vol.3, no., pp.2223$2228,2000$.

[5] Lima, J.L., Goncalves, J.C., Costa, P.G., Moreira, AP., "Humanoid robot simulation with a joint trajectory optimized controller,", Emerging Technologies and Factory Automation, 2008. ETFA 2008. IEEE International Conference on, vol., no., pp.986,993, 15-18, Sept. 2008.

[6] Zhibin Li., Tsagarakis, N.G., Caldwell, D.G., "Walking trajectory generation for humanoid robots with compliant joints: Experimentation with COMAN humanoid,", Robotics and Automation (ICRA), 2012 IEEE International Conference on , vol., no., pp.836,841, 14-18, May 2012.

[7] Wenqi Hou., Jian Wang., Jianwen Wang., Hongxu Ma., "Online Biped Walking Pattern Generation with Contact Consistency", IAES International Journal of Robotics and Automation (IJRA), Vol 4, No 1, March 2015.

[8] Young-Jae Ryoo., "Development of Child-Sized Humanoid Robot for Dance Performance", Advances in Intelligent Systems and Computing, vol. 274, pp. 725-732, 2014.

[9] Fatemeh Maleki., Zeinab Farhoudi., "Making Humanoid Robots More Acceptable Based on the Study of Robot Characters in Animation", IAES International Journal of Robotics and Automation (IJRA), vol 4, No 1, March 2015.

[10] Park, Ill-Woo., et al., "Mechanical design of the humanoid robot platform, HUBO", Journal of Advanced Robotics, 21.11 (2007): 1305-1322.

[11] James, J., Kuffner, Jr., Satoshi Kagami., Koichi Nishiwaki,, Masayuki Inaba., Hirochika Inoue., "DynamicallyStable Motion Planning for Humanoid Robots", Journal of Autonomous Robots, Volume 12, Issue 1, pp 105-118, Jan 2002.

[12] Pin-Yong Ling., Chao, K.Y.-W., Han-Pang Huang., Jiu-Lou Yan., "Footprint searching and trajectory design of a humanoid robot", Robotics and Biomimetics (ROBIO), 2012 IEEE International Conference on , vol., no., pp.259,264, 11-14 Dec. 2012.

[13] Dingsheng Luo., Yi Wang., Xihong Wu., "Online learning of COM trajectory for humanoid robot locomotion" ,Mechatronics and Automation (ICMA), 2012 International Conference on , vol., no., pp.1996,2001, 5-8 Aug. 2012.

[14] Dong W. Kim,Nak-Hyun Kim,Gwi-Tae Park, "ZMP based neural network inspired humanoid robot control," Journal of Nonlinear Dynamics, Volume 67, Issue 1, pp 793-806, January 2012.

[15] W Yu, Y Ikemoto, R Acharya, J Unoue, "Comparing normal walking and compensated walking: their stability and perturbation resistance. A simulation study," Proceedings of The Institution of Mechanical Engineers, Part H: Journal of Engineering in Medicine, vol 224, pp 891-901, 2010.

[16] MIOMIR VUKOBRATOVIĆ, BRANISLAV BOROVAC, "ZERO-MOMENT POINT — THIRTY FIVE YEARS OF ITS LIFE", International Journal of Humanoid Robotics, Volume 01, Issue 01, March 2004.

[17] Erbatur, K., Okazaki, A., Obiya, K., Takahashi, T., Kawamura, A., "A study on the zero moment point measurement for biped walking robots," Advanced Motion Control, 2002. 7th International Workshop on, vol., no., pp.431,436, 2002.

[18] Jung-Yup Kim., Ill-Woo Park., Jun-Ho Oh., "Experimental realization of dynamic walking of the biped humanoid robot KHR-2 using zero moment point feedback and inertial measurement", Journal of Advanced Robotics, vol 20, Issue 6, pages 707-736, 2006.

[19] Kajita, S., Morisawa, M., Harada, K., Kaneko, K., Kanehiro, F., Fujiwara, K., Hirukawa, H., "Biped Walking Pattern Generator allowing Auxiliary ZMP Control", Intelligent Robots and Systems, 2006 IEEE/RSJ International Conference on , vol., no., pp.2993,2999, 9-15 Oct. 2006. 